\title{
Derivation of Strategic Logistic Measures for Forging Systems
}

\author{
Peter Nyhuis, Felix S. Wriggers and Tim D. Busse \\ Institute of Production Systems and Logistics, \\ Gottried Wilhelm Leibniz Universität Hannover, \\ An der Universität 2, D-30823 Garbsen, Germany \\ e-mail: wriggers@ifa.uni-hannover.de
}

\begin{abstract}
In order to thrive in global markets, enterprises have to distinguish themselves from their competitors not only by manufacturing high quality products at low costs, but also with superior logistic performance. Logistic Operating Curves (LOC) can be applied to facilitate this as well as to derive strategic measures. This will be demonstrated based on the example of the German forging industry.
\end{abstract}

\section{Keywords}

Logistic Operating Curves, logistic measures

\section{Introduction}

A superior logistic performance is primarily demonstrated by high delivery reliability and short delivery times [1], [2], [3]. The criticalness of superior logistic performance is particularly noticeable in the German forging industry. Companies in this sector are usually small and medium-sized enterprises (SME) delivering about $70 \%$ of the forged parts directly to automotive OEMs [4], [5]. They thus tend to be integrated in just-in-time and just-in-sequence delivery concepts [6], [7].

Short throughput times and a high level of schedule reliability in production are a prerequisite for accomplishing short delivery times and a high level of delivery reliability. In contrast, low costs require a high output rate [8] whereas the logistic objectives throughput time and output rate, oppose each other: While short throughput times can only be realized with a low work in process (WIP) level, high output rates depend on high WIP levels. This conflict between logistic objectives is commonly known as the 'Dilemma of Operations Planning' [9]. 
A solution for controlling this dilemma is offered by the Logistic Operating Curve Theory (LOC Theory) developed at the Institute of Production Systems and Logistics, Leibniz University of Hanover [10].

\section{Modeling Logistic Processes in the Forging Industry}

The starting point for developing all of the sub-models for the Logistic Operating Curve Theory was defining a fundamental production logistics' element - the throughput element. Based on the logistic process levels, the throughput element defines the throughput time of an operation as the time span which an order requires from the ending of the preceding process or from the point in time of the order's input up to the end of the processing in the observed operation. When manufacturing is conducted according to lots, as is common in the forging industry, an order is transported to the following workstation after it is processed or if necessary after a period of waiting. Usually the lot then meets up with orders that are already queued and therefore has to wait until those before it are processed. Initially it is required that the orders are completed according to First In First Out (FIFO). As long as the capacities for processing the orders are available, the station's set-up can be changed and the next lot can be processed. This cycle continues until the order has passed through all the required operations.

\subsection{The Funnel Model and Throughput Diagram}

With the help of the throughput elements definition, the throughput time, scheduling deviation and their components can be calculated and statistically evaluated. The throughput element also forms the basis for the Funnel Model and the Throughput Diagram derived from it (Figure 1).

With the Funnel Model, similarly to diagrams of flow process methods, the throughput behavior of every random capacity unit in a manufacturer can be completely described through the input, WIP, and output (Figure la). Together with those already waiting the lots arriving at the workstation form a store of waiting orders. Once they are processed they flow out the funnel. The funnel opening thus symbolizes the station's output rate, which can vary within the capacity limits.

The funnel's events can be transferred to the so-called "Throughput Diagram" (Figure $1 \mathrm{~b}$ ). The completed orders are cumulatively plotted with their work content over the completion schedule (output curve). The input curve is developed similarly, in that the incoming orders are plotted with their work content over the input schedule. The start of the input curve is determined by the WIP, which is found on the workstation at the start of the investigation period (initial WIP). At the end of the investigation period, the end WIP can be read from the diagram. Whereas, the mean slope of the input curve corresponds to the mean load, the output curve's mean slope corresponds to the mean output rate. 


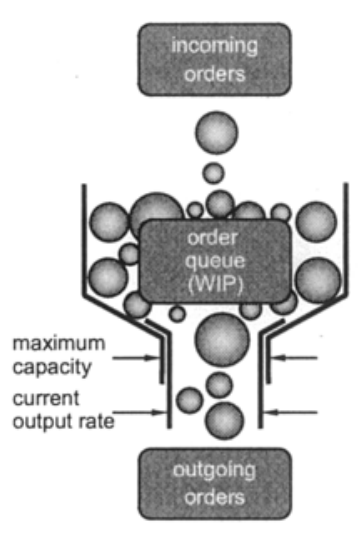

a) Funnel Model

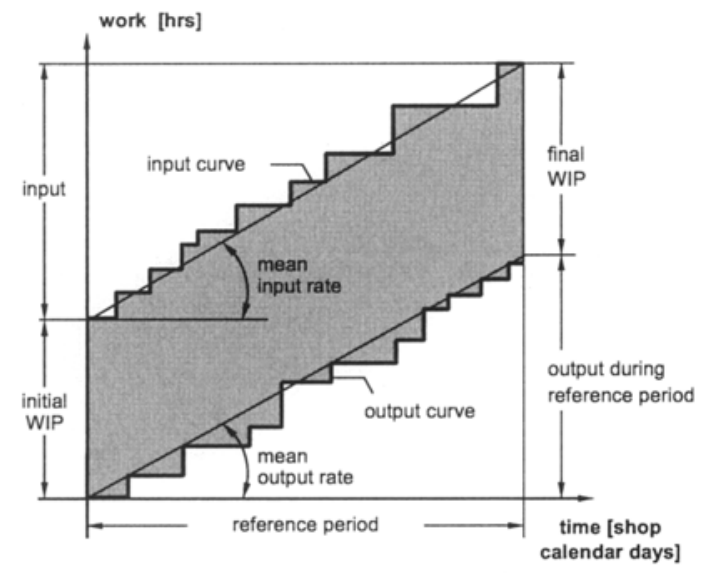

b) Throughput Diagram

Fig. 1. The Funnel Model and Throughput Diagram

The Throughput Diagram describes the dynamic system behavior completely quantitatively and exactly with regards to time. It also provides support in identifying causes of plan deviations as well as for deriving control measures. When necessary the Throughput Diagram can be supplemented by diagrams of the throughput time, work content and/or distribution of the scheduling deviations. Fundamental information about the production's processes can thus be gained and analyzed with regards to a variety of problems. Nevertheless, these do not explain or only partially explain the interactions between the logistic parameters.

The following described Logistic Operating Curves (LOC) however, provide support in understanding cause and effect relations between these.

\subsection{Logistic Operating Curves}

The Funnel Model, Throughput Diagram and figures derived from them each describe a specific relaxed operating state. In the upper part of Figure 2, three basically different operating states are depicted in simplified Throughput Diagrams. These operating states can now be strongly aggregated in the form of Logistic Operating Curves. In order to do so, the relevant values for the output rate and range are plotted as a function of the corresponding WIP level. The Output Rate Operating Curve (OROC) clearly illustrates that a workstation's output rate does not significantly change beyond a specific WIP value. There is a continuous queue of work and thus no WIP related interruptions in the processing. Below this WIP value however there are increasing output setbacks due to a temporary lack of queued orders. The range (and with that the throughput time) though, increases above the critical WIP value for the most part proportionally to the WIP. Nonetheless, when the WIP is reduced both throughput parameters cannot fall below a specific 
minimum. For the throughput time, this minimum results from the mean operation time for the orders and where applicable the transport time.

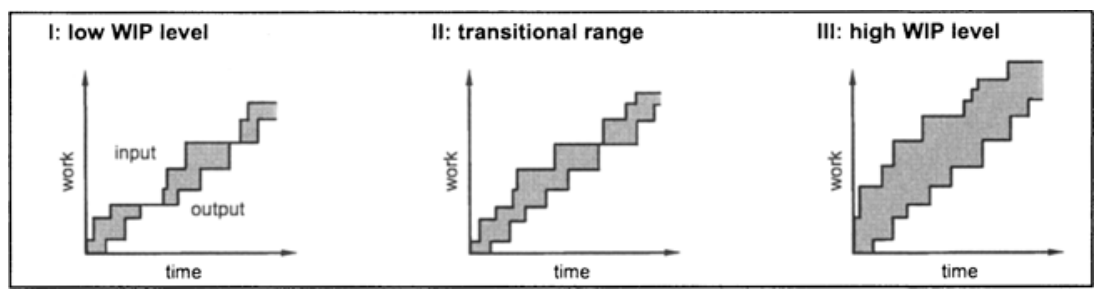

a) typical operating states for a workstation

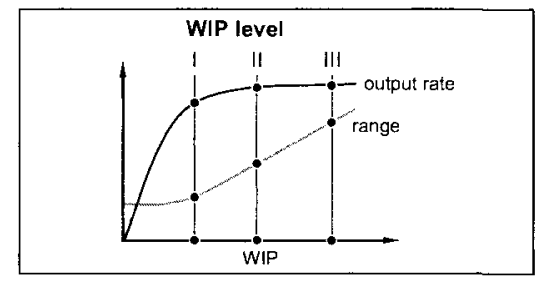

b) depiction of operating states in Logistic Operating Curves

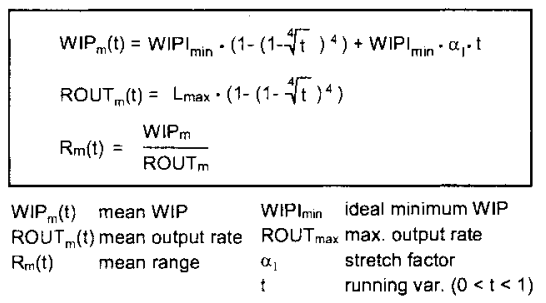

c) approximation equations for calculating the Logistic Operating Curves

Fig. 2. Deriving the Logistic Operating Curves

A momentary state on a workstation always corresponds to only one operating state and thus one operating state point on a LOC. The Logistic Operating Curves represent how the observed system behaves when a different WIP level is set given otherwise stable boundary conditions. They thus characterize the logistic behavior of a production when the WIP changes. Beyond that, it is also possible to develop LOC for changed manufacturing or order structures and to compare these with one another. In this way, the impact of intervening in the production process can be evaluated with regards to logistic aspects.

It can easily be seen that the basic form of the Logistic Operating Curves are applicable to every arbitrary production system: WIP reductions lead to decreased throughput times but also under certain circumstances to breaks in the material flow and thus to utilization losses. However, the specific shape of the LOC for the observed workstations are dependent on different boundary conditions such as the capacity, the orders to be processes (in particular the mean value and their distribution) and how they are incorporated into the system's material flow. A detailed description of the Logistic Operating Curve Theory, the influences and the basic equations can be found in NYHUIS and WIENDAHL [10].

Logistic Operating Curves thus allow the dependencies between the logistic objectives utilization, throughput time, schedule reliability and WIP to be expressed [11]. At the same time, the cost-benefit ratio of applying the LOC is extremely favorable [12]. Due to this they are extremely well suited for increasing an 
enterprises logistic performance [13] and have therefore been widely received and accepted both in research and on the production floor [10].

\section{Fields of Application for Logistic Operating Curves}

Logistic Operating Curves represent an ideal basis for developing and monitoring an enterprise's process reliability and capability [13]. LOC can thus be drawn upon for evaluating processes during a production control. They indicate for example which throughput times and WIP levels can be achieved on a workstation with the existing structural conditions, without having to expect appreciable breaks in the material flow and resulting output losses. When applying them within production planning and control (PPC), system parameters can be derived and set which conform with the goals. Depicting the logistic objectives in a diagram makes it possible to decide which attribute should be weighted the most depending on the current market and operational situations as well as the workstation's specific boundary conditions. Simultaneously it can be shown, how changing a parameter impacts the logistic attributes. Instead of searching for an imaginary optimum, a primary, frequently market dependent objective e.g. a desired throughput time, is assumed. The remaining target values such as the output rate and WIP inevitably result from there.

If during the application it turns out that the set target values are not achievable without further intervening measures which change the form of the LOC, then the Logistic Operating Curves can be used to support and evaluate the planning activities corresponding to the imagined possibilities. Alternative planning and control strategies can therefore be evaluated and chosen based on logistic criteria. The LOC Theory can also be directly integrated into PPC such as in methods for determining lot size, scheduling or also for the order release. Moreover, it supports a continual, method based, alignment of the planning and controls with the logistic objectives. During the planning stage of a factory the Logistic Operating Curves can be drawn upon for a logistic oriented evaluation of alternative manufacturing principles or new logistic concepts. Furthermore, it is also possible to evaluate investment decisions (e.g. implementing new transport systems, introducing new production technology) and to extend the modeling of business processes.

Since the LOC express the cause and effect relations between the logistic objectives output rate, throughput time, and WIP both qualitatively and quantitatively, they offer effective support for mastering the 'dilemma of operations planning'. Based on these and depending on the actual operating state - which can also differ from place to place - it can be decided which objective should be assigned the greatest significance.

Prioritizing a goal forms a basis on which the targeted operating points, defined through the WIP, output rate and throughput time can be determined for the individual workstations. Within this process the existing structures for the work content and capacities on the one hand, as well as both the required delivery date, required capacities and the cost structures on the other hand are considered. In the following this procedure will be referred to as a "Logistic Positioning". The Logistic 
Positioning is the basis for all of the mentioned applications, it establishes the targets and thus represents a link between all of the individual functions [10].

In addition the determined target values and if necessary the allowable fields of tolerance can be directly checked for consistency. If the throughput time is given for a workstation, the corresponding key figures for the output rate, utilization and WIP result directly from it.

By conducting such a positioning it can be shown whether or not the targeted goals are realistic with the existing boundary conditions. If it is not the case, the target values are not located on the calculated Logistic Operating Curves. Measures can then be taken in order to develop new logistic potential, that is measures which influence the behavior of the Logistic Operating Curves [10].

\section{Developing a Method for Choosing Strategic Measures}

During the Logistic Positioning, values for the four logistic objectives (WIP, scheduling reliability, utilization and throughput time) are recorded. The actual and target values for these parameters can be formulated as a four dimensional Logistic Position Vector (LPV). The difference between the LPV for the target state and that of the actual state defines the so called Logistic Target Achievement Vector (LTV). Based on the actual state, the Logistic Target Achievement Vector describes the changes required for the production logistic objectives in order to attain the target state.

In order to realize the target state, strategic measures have to be taken. Generally though, there will be a number of both complementary and conflicting possible measures. Decision makers in an enterprise perceive themselves as being confronted with the problem of which measures are best suited for the specific situation. In order to answer this question, a method is currently being developed at the Institute of Production Systems and Logistics, which permits a monetary evaluation of the measures and thus forms the basis of a decision support system (DSS) for choosing strategic measures.

This method requires a description of the functional correlations between the measures and their monetary impact, which can be determined based on positive and negative payment stream parameters. Up until now, the functional correlation between the measures for improving the production logistics and the payment stream parameters was missing. Monetary transfer functions were thus developed. On the one hand, monetary transfer functions produce the correlation between the measures and the revenues and on the other hand, the correlation between the measures and the costs. 

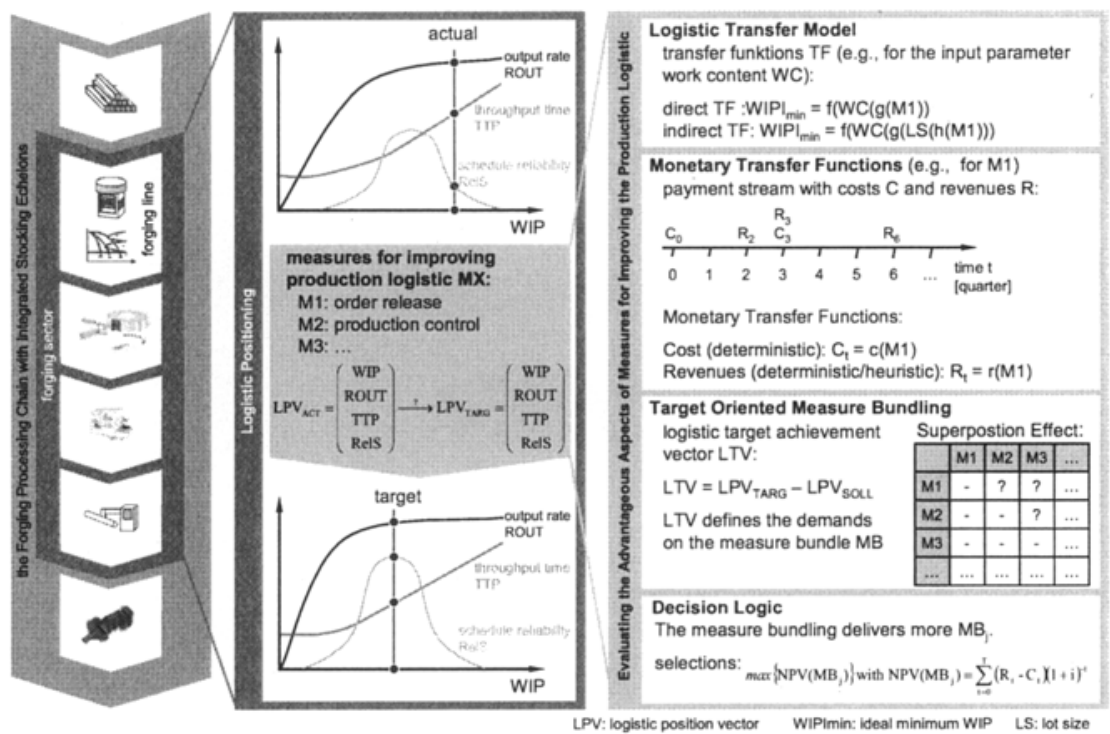

Fig. 3. Expanding the Logistic Operating Curve Theory in order to Derive Strategic Logistic Measures

Evaluating and choosing the measures, should thus occur based on standard economical parameters such as the capital value, the return on investment or the EVA (economic value added).

\section{Acknowledgements}

The authors would like to thank the German Research Foundation (DFG) for financially supporting the work described in this article as part of the sub-project C2 of the interdisciplinary research center 489 "Processing Chains for the Production of Precision Forged High Performance Components".

\section{References}

1. Hon, K. K. B., Performance and Evaluation of Manufacturing Systems, Annals of the CIRP. Volume 55, Number 2 (2005).

2. Enslow, B., Best Practices in International Logistic (Aberdeen Group: Boston 2006).

3. Nyhuis, P., Wriggers, F., Fischer, A., The International Federation of Information Processing (IFIP), Volume 207, Knowledge Enterprise: Intelligent Strategies in Product Design, Manufacturing, and Management, eds. K. Wang, Kovacs, G., Wozny, M., Fang, M. (Boston: Springer 2006).

4. Rudau, E., Deutsche Gesenkschmiedeindustrie gerüstet für die Zukunft, Schmiede-Journal. (2000). 
5. Hirschvogel, M., Schmiedetechnik in Europa und in den USA - technischer oder kultureller Unterschied, Schmiede-Journal (2001) pp. 34-36.

6. Wiendahl, H.-P.; Ruta, A., Einsatz der Logistik FMEA, Schmiede-Journal (1999), pp. 39 40.

7. Boston Consulting Group: Steering Carmaking into the 21st Century - From today's best practices to the transformed plants of 2020 (BCG:Boston 2001).

8. Lödding, H., Verfahren der Fertigungssteuerung - Grundlagen, Beschreibung, Konfiguration (Springer:Berlin 2005).

9. Gutenberg, E., Grundlagen der Betriebswirtschaftslehre, Volume 1 - Die Produktion, (Springer:Berlin 1951).

10. Nyhuis P., Wiendahl H.-P., Fundamentals of Production Logistics. Transl. of 3rd ed. (To be published Springer:Berlin 2007).

11. Nyhuis, P., Logistic Production Operating Curves - Basic Model of the Theory of Logistic Operating Curves, Annals of the CIRP, Volume 55, Number 1 (2006) pp. 441-444.

12. Nyhuis, P., von Cieminski, G., Fischer, A., Applying Simulation and Analytical Models for Logistic Performance Prediction, Annals of the CIRP, Volume 54, Number 1 (2005) pp. 417-422.

13. Wiendahl, H.-P.: Collaborative Supply Chain Planning - A Case Study from the German Cutting Tool Industry, Collaborative Systems for Production Management eds. Jagdev, $\mathrm{H}$. S., Wortmann, J. C., Pels, H. J (Kluwer:Norwell 2003). 